\title{
Male Patients with Longstanding Type 2 Diabetes Have a Higher Incidence of Hypoglycemia Compared with Female Patients
}

\author{
Feng-fei Li · Ying Zhang • Wen-li Zhang - Xiao-mei Liu • \\ Mao-yuan Chen · Yi-Xuan Sun · Xiao-fei Su • Jin-dan Wu • \\ Lei Ye $\cdot$ Jian-hua Ma (iD
}

Received: July 10, 2018 / Published online: August 22, 2018

(C) The Author(s) 2018

\section{ABSTRACT}

Introduction: To explore whether there was a gender difference in the risk of hypoglycemia during intensive insulin therapy in patients with longstanding type 2 diabetes (T2D). This was a post hoc analysis of a single-center, openlabel and prospective trial.

Methods: All subjects were admitted as inpatients, underwent a standard bread meal test at baseline and received a 7-day continuous subcutaneous insulin infusion (CSII) therapy for achieving glycemic control. Patients then were

Feng-fei Li, Ying Zhang, Wen-li Zhang, Xiao-mei Liu, Mao-yuan Chen and Yi-xuan Sun contributed equally to this article.

Enhanced digital features To view enhanced digital features for this article, go to https://doi.org/10.6084/ m9.figshare.6965816.

F. Li · Y. Zhang - W. Zhang - X. Liu - M. Chen .

Y. Sun $\cdot$ X. Su $\cdot$ J. Wu $\cdot$ J. Ma $(\bowtie)$

Department of Endocrinology, Nanjing First

Hospital, Nanjing Medical University, Nanjing,

China

e-mail: majianhua196503@126.com

L. Ye

National Heart Research Institute Singapore,

National Heart Centre Singapore, Singapore,

Singapore randomized 1:1 to two groups receiving (1) 4 days of Novo Mix 30 followed by 2 days of Humalog Mix 50; (2) 4 days of Humalog Mix 50 followed by 2 days of Novo Mix 30. All patients were subjected to 4-day retrospective continuous glucose monitoring (CGM) during the last 4 days in this study. The primary outcome was the incidences of hypoglycemia monitored by CGM at the end point.

Results: A total of 102 patients met the inclusion criteria and completed the study. Our data revealed that 29 patients (28\%) experienced hypoglycemia as detected by CGM at the end point. Binary logistic stepwise regression analysis showed that only gender significantly correlated with hypoglycemia ( $B=1.17$, $p=0.017)$. Importantly, male patients had a significantly higher incidence of hypoglycemia than female patients (male $=20 / 52$, female $=9 /$ $50, p=0.022$ ), although male patients required significantly lower insulin doses to maintain glycemic control than female patient $(p=0.00)$. Conclusion: Male patients with longstanding T2D had a higher incidence of hypoglycemia than female patients during intensive insulin therapy.

Trial registration: ClinicalTrials.gov identifier, ChiCTR-IPR-15007340.

Keywords: Hypoglycemia; Insulin therapy; Male; Type 2 diabetes 


\section{INTRODUCTION}

Several studies indicate that gender differences play a role in risk, progression, prevention and glucose-lowering therapy for type 2 diabetes (T2D) [1-3]. We conducted a retrospective study using continuous glucose monitoring (CGM) showing that older males with newly diagnosed T2D have a higher risk of nocturnal hypoglycemia during intensive insulin therapy compared with older females [4]. Therefore, a better understanding of gender-sensitive clinical approaches is imperative [5].

Continuous subcutaneous insulin infusion (CSII) is an effective therapeutic for treating hyperglycemia when conventional therapies are no longer sufficient to maintain glycemic control in T2D patients [6, 7]. Early administration of a CSII therapy leads to a significant improvement in beta cell function and prolonged remission in newly diagnosed T2D patients [8-11]. Even in longstanding T2D patients, CSII therapy is still able to further decrease the A1c levels [12]. Using CGM, we also observed that CSII provided a greater improvement in glycemic variations in both newly diagnosed T2D and longstanding T2DM patients [6].

However, hypoglycemia, especially nocturnal hypoglycemia, is a side effect of intensive insulin therapy [13], which may be an important barrier for patients with diabetes trying to achieve better glucose control as it may result in sudden death in severe cases [14]. Nearly $20 \%$ of patients using insulin pump therapy and onefifth of patients had symptomless nocturnal hypoglycemia [15]. Studies further highlighted that older patients had high incidences of hypoglycemia [16, 17], although we observed that newly diagnosed male and female T2D patients required similar insulin doses to maintain glycemic control [18]. The gender differences in insulin doses required to maintain euglycemic control in patients with longstanding T2D remain largely unknown.

In this study, we tried to clarify whether there was a gender difference in hypoglycemia during intensive insulin therapy in patients with longstanding T2D.

\section{METHODS}

This was a post hoc analysis of a single-center, open-label and prospective trial, which focused on patients with T2D to determine whether patients would benefit from premixed insulin therapy after CSII for achieving glycemic control (ClinicalTrials.gov, no. ChiCTR-IPR15007340). The study protocol and patient consent forms were approved by the Institutional Ethics Committee of Nanjing First Hospital, Nanjing Medical University. All procedures were in accordance with the ethical standards of Nanjing First Hospital and with the Helsinki Declaration of 1964 as revised in 2013. Informed consent was obtained from all patients recruited in the study.

Between February 2013 and December 2014, we recruited a total of 118 patients with T2D who failed oral antihyperglycemic agents in Nanjing First Hospital, Nanjing Medical University. The inclusion criteria were (1) patients aged between 18 and 80 years; (2) 7.5\% $\leq \mathrm{HbA} 1 \mathrm{c} \leq 12 \%$. Patients were excluded from analysis if they were positive for antiglutamic acid decarboxylase antibody or if they had maturity onset diabetes in the young (MODY) or mitochondrial diabetes mellitus [7].

At baseline, all subjects were admitted as inpatients and underwent a standard bread meal test (100 g) [19]. Serum samples were obtained at 0, 30 and 120 min after meal loading. Serum C-peptide and glucose concentrations were measured centrally at the central laboratory in Nanjing First Hospital, Nanjing Medical University. Then, recruited subjects received 7-day CSII therapy to achieve glycemic control. CSII therapy was provided with aspart (Novo Nordisk, Bagsvaerd, Denmark) using a Medtronic insulin pump (Northridge, CA) as previously described $[6,18,20]$. After subjects achieved glycemic control, defined as a fasting and 2-h post prandial capillary blood glucose concentration between 6.1 and $8.0 \mathrm{mmol} / \mathrm{l}$ [21], patients were randomized 1:1 to two groups receiving: (1) 4 days of Novo Mix 30 (Novo Nordisk, Bagsværd, Denmark) followed by 2 days of Humalog Mix 50 (humalog ${ }^{\circledR} \mathrm{Mix}^{\mathrm{TM}}{ }^{\mathrm{TM}}$, Eli Lilly and Co., Indianapolis, IN, USA); (2) 
4 days of Humalog Mix 50 followed by 2 days of Novo Mix 30. Initial premixed insulin doses were calculated as $0.4-0.5 \mathrm{IU} / \mathrm{kg}$, and doses were subsequently adapted according to capillary glucose values obtained by self-monitoring during the first 2 days of the premixed insulin titration period. Investigators titrated insulin doses on an individual patient basis with the titration algorithm (if the blood glucose level was $<4.4 \mathrm{mmol} / \mathrm{l}$, the basal insulin dose was reduced 2 units; if the blood glucose level was within $4.4-6.1 \mathrm{mmol} / \mathrm{l}$, the basal insulin dose was unchanged; if the blood glucose level was within $6.2-7.8,7.9-10.0$ and $>10.0 \mathrm{mmol} / \mathrm{l}$, the basal insulin dose was increased subsequently by 2,4 and 6 units, respectively), as described previously $[6,22]$. Therapy was then unchanged for another 4 days, including 2 days of Humalog Mix 50 and 2 days of Novo Mix 30 after glycemic control had been achieved. During the premixed insulin treatment period, patients did not take any oral antihyperglycemic agents.

All patients were subjected to 4-day retrospective CGM (Sof-sensor, CGMS-Gold, Medtronic Incorp., Northridge, CA, USA) at the last 4 days in this study $[18,23]$. All subjects were instructed to maintain moderate physical activity and received breakfast (0700), lunch (1100) and dinner (1700). The total daily caloric intake and percentages of carbohydrate, proteins and fats of each meal were the same as previously described [24].

We analyzed the day 2 and day 4 CGM data to compare the glycemic profiles between the two premixed insulin groups in this study. Because of the premixed insulin cross-over design, at least a 24 -h washout period was needed [25]. The 24-h mean glucose concentrations (MGs), 24-h mean amplitude of glycemic excursions (MAGE), percentage time duration (\%) of hyperglycemia (defined as glucose concentration $>10.0 \mathrm{mmol} / \mathrm{l}$ ) and hypoglycemia (defined as glucose concentration $<3.9 \mathrm{mmol} /$ l) $[4,22]$, incremental area under the curve (AUC) of hyperglycemia and incremental area over the curve (AOC) were calculated by software from Medtronic Incorp. (USA), and the hypoglycemiic episodes were also recorded. $\beta$ Cell function was assessed by homoeostasis model assessment B (HOMA-B), the insulin sensitivity was indicated by the homoeostasis model assessment insulin resistance (HOMA-IR) $[7,26]$, and the Matsuda Index was calculated as previously described $[27,28]$. The insulin doses of the CSII and Novo Mix 30 therapy period were recorded, respectively.

The primary outcome was the incidences of hypoglycemia at the end point. The secondary end points were the differences in insulin doses and glycemic profiles between the two groups.

\section{Statistical Analysis}

All data are presented as the means \pm SD. Statistical analysis was performed using SPSS software (version 17.0; SPSS, Inc., Chicago, IL, USA). Shapiro-Wilk test was used to assess the distribution of data. A chi-squared test was performed comparing the ratio differences between the two groups. The mixed ANOVA model $(2 \times 2)$ test was used to compare differences between groups. A two-way ANOVA was used for repeated measurements for the group comparisons followed by the Bonferroni-Dunn post hoc test. We used correlation and binary logistic stepwise regression analyses to examine the interrelationships between gender and hypoglycemia. $P$ values were two tailed with a significance level of $5 \%$.

\section{RESULTS}

A total of 118 patients were recruited into this study. Seven patients who had glucose levels $>$ $22.2 \mathrm{mmol} / \mathrm{l}$ during the CGM period were excluded as the CGM sensor used in this study was unable to monitor glucose concentrations $>22.2 \mathrm{mmol} / \mathrm{l}$, and 9 patients did not meet the inclusion criteria (Fig. 1). The 102 patients who completed the study with age $59.4 \pm 11.8$ years, body mass index (BMI) $23.0 \pm 7.3 \mathrm{~kg} / \mathrm{m}^{2}, \mathrm{HbA}_{1 \mathrm{c}}$ values $9.8 \pm 2.6 \%$ and course of disease $9.4 \pm 5.7$ years were analyzed.

We analyzed the incidence of hypoglycemia between groups at the end point. Our data revealed that 29 (28\%) patients experienced hypoglycemia as detected by CGM. Correlation analysis showed that BMI, age, course of disease, 


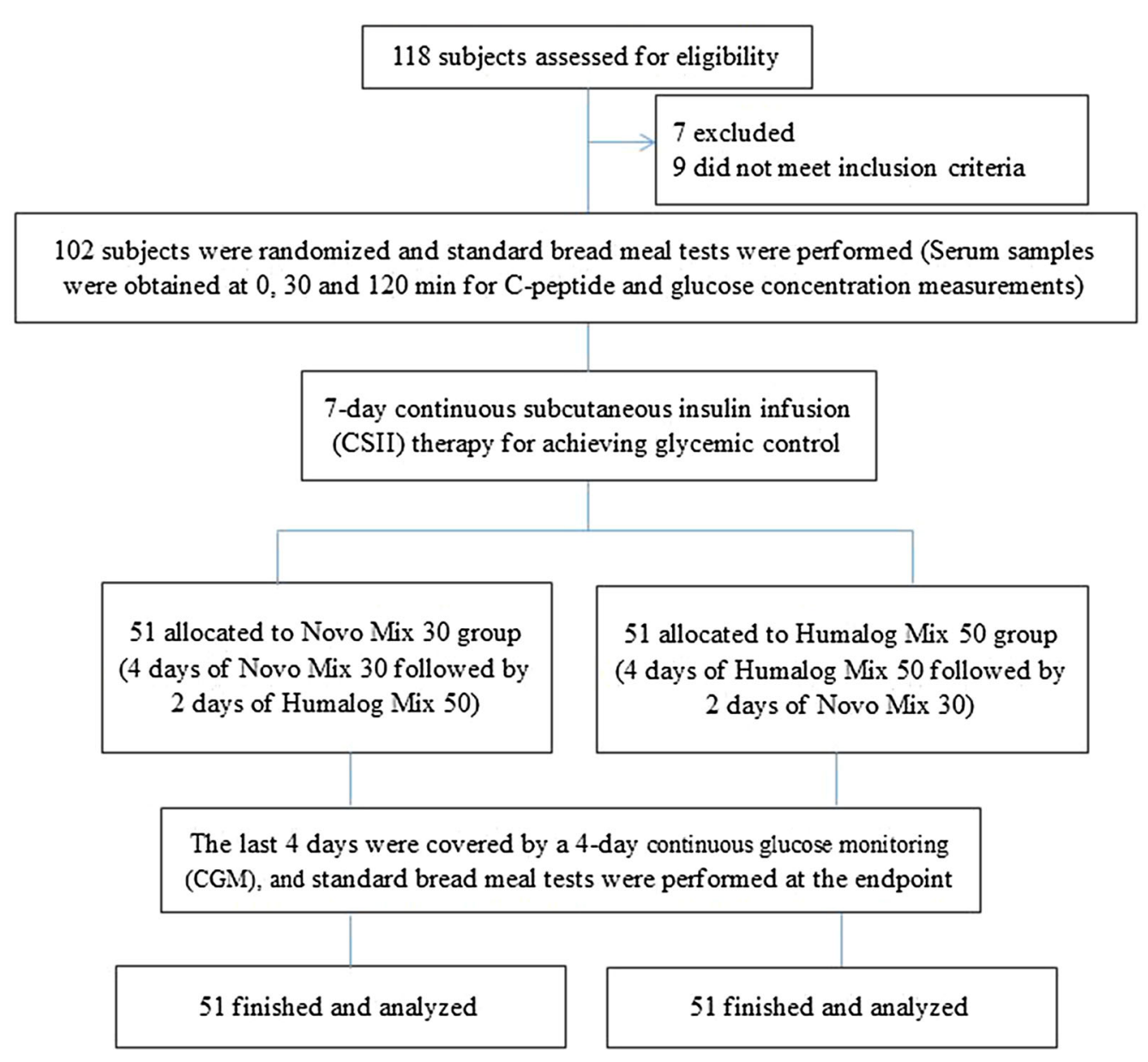

Fig. 1 Study flow chart

HbA1c values and gender were correlated with hypoglycemia, respectively. We performed a binary logistic stepwise regression analysis, controlling for BMI, age, course of disease, HbA1c values and gender, to identify which factors contributed to the increased incidences of hypoglycemia. Interestingly, only gender was the independent factor for hypoglycemia $(B=1.17, \quad p=0.017)$. Furthermore, a chisquared test was performed to compare the hypoglycemic ratio differences between patients in the male and female groups. Our data indicated that males had a higher incidence of hypoglycemia compared with females ( males $=20 / 52$, females $=9 / 50 ; p=0.022$ ).

We therefore analyzed the gender differences in glycemic variations at the end point. We did not observe any difference in hourly blood glucose concentrations between the male and female patients (Fig. 2). Our CGM data showed that male and female patients had similar 24-h MG, SDBG, MAGE, percentage of time duration (\%) of hyperglycemia and hypoglycemia, incremental AUC of hyperglycemia and the incremental AOC of hypoglycemia (Table 1).

There were no differences in the mean values of various measurements between male and female groups, such as age $(p=0.47)$, BMI $(p=0.76)$, waistline $(p=0.64)$, waist-to-hip ratio $(p=0.55)$, course of disease $(p=0.63)$ and HbA1c values $(p=0.99)$ (Table 2$)$.

Male and female patients took similar time to achieve glycemic control during CSII therapy $(6.1 \pm 3.7$ vs. $6.6 \pm 3.7$ days, $p=0.52)$. Our results surprisingly showed lower total, basal and bolus insulin doses $(p<0.01$, respectively) in the male than female patient group during the CSII period. We also analyzed the insulin 


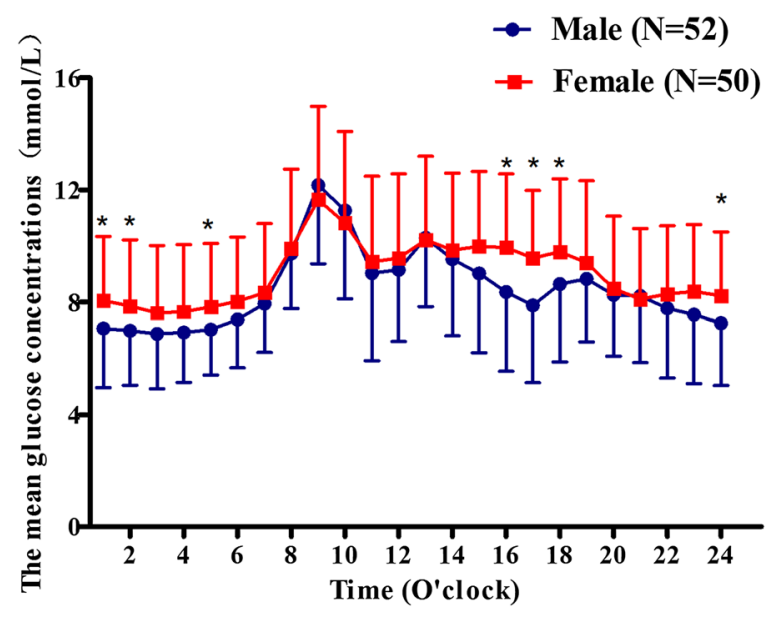

Fig. 2 Hourly glucose concentrations in male and female patients

doses required by patients between the two groups at the end point. Our data showed that male patients required significantly lower premixed insulin doses $(p=0.00)$, with a significant reduction in breakfast $(p=0.01)$, lunch $(p=0.02)$ and dinner insulin doses $(p=0.00)$ to maintain glycemic control at the end point than female patients (Table 3 ).
Table 2 Baseline of male and female patients with type 2 diabetes

\begin{tabular}{llll}
\hline Items & Male group & $\begin{array}{l}\text { Female } \\
\text { group }\end{array}$ & $\boldsymbol{p}$ value \\
\hline Number & 52 & 50 & - \\
Age (years) & $58.6 \pm 11.7$ & $60.3 \pm 12.0$ & 0.47 \\
Duration & $6.2 \pm 6.3$ & $7.0 \pm 6.2$ & 0.63 \\
BMI & $22.8 \pm 6.4$ & $23.3 \pm 8.2$ & 0.76 \\
HbAlc & $9.8 \pm 3.0$ & $9.8 \pm 2.1$ & 0.99 \\
Waistline & $78.7 \pm 26.8$ & $75.9 \pm 32.6$ & 0.64 \\
Waist-to-hip & $0.8 \pm 0.3$ & $0.8 \pm 0.3$ & 0.55 \\
$\quad$ ratio & & & \\
\hline
\end{tabular}

Data are presented as means $\pm \mathrm{SD}$

${ }^{*} p<0.05$. Age (years); Duration (years): duration of T2D; BMI: body mass index $\left(\mathrm{kg} / \mathrm{m}^{2}\right)$, HbAlc: glycated hemoglobin (\%); waistline $(\mathrm{cm})$; waist-to-hip ratio

To analyze the mechanism underlying the different insulin dose required to maintain glycemic control for male and female patients, we compared $\beta$ cell function and insulin resistance between the two groups using the HOMA-B and

Table 1 Glycemic variations in male and female patients with type 2 diabetes

\begin{tabular}{llll}
\hline Items & Male group & Female group & $\boldsymbol{p}$ value \\
\hline Number & 52 & 50 & $/$ \\
24-h MG & $8.5 \pm 1.5$ & $9.0 \pm 1.8$ & 0.08 \\
SDMG & $2.3 \pm 0.8$ & $2.2 \pm 0.7$ & 0.58 \\
MAGE & $5.8 \pm 2.2$ & $5.3 \pm 2.2$ & 0.28 \\
Duration above & $27.1 \pm 22.0$ & $34.4 \pm 24.5$ & 0.12 \\
Duration below & $3.2 \pm 5.4$ & $0.9 \pm 2.8$ & 0.01 \\
AUC $>10.0 \mathrm{mmol} / \mathrm{l}$ & $0.6 \pm 0.7$ & $0.8 \pm 0.8$ & 0.17 \\
AUC $<3.9 \mathrm{mmol} / \mathrm{l}$ & $0.1 \pm 0.0$ & $0.0 \pm 0.0$ & 0.17 \\
\hline
\end{tabular}

Data are presented as means $\pm \mathrm{SD}$

24-h $M G$ 24-h mean glucose concentrations (mmol/l), SDMG 24-h standard deviation of mean glucose concentrations (mmol/l), MAGE 24-h mean amplitude of glycemic excursions (mmol/l), Duration above duration $>10 \mathrm{mmol} / \mathrm{l}$ (\%), Duration below duration $<3.9 \mathrm{mmol} / \mathrm{l}(\%), A U C>10.0 \mathrm{mmol} / \mathrm{l}$ incremental AUC of glucose level $>10.0 \mathrm{mmol} / \mathrm{l}(\mathrm{mmol} /$ l day), $A U C<3.9 \mathrm{mmol} / \mathrm{l}$ the incremental AUC of glucose $<3.9 \mathrm{mmol} / \mathrm{l}(\mathrm{mmol} / \mathrm{l}$ day)

${ }^{*} p<0.05$ 
Table 3 Insulin doses and glycemic variations in male and female patients with type 2 diabetes

\begin{tabular}{llll}
\hline Items & Male group & Female group & $\boldsymbol{p}$ value \\
\hline TDD1 & $0.55 \pm 0.22$ & $0.73 \pm 0.26$ & $0.00^{*}$ \\
Basal & $0.26 \pm 0.11$ & $0.37 \pm 0.16$ & $0.00^{*}$ \\
Bolus & $0.29 \pm 0.11$ & $0.37 \pm 0.16$ & $0.01^{*}$ \\
TDD2 & $0.54 \pm 0.22$ & $0.70 \pm 0.25$ & $0.00^{*}$ \\
BI & $0.22 \pm 0.10$ & $0.27 \pm 0.09$ & $0.01^{*}$ \\
LI & $0.15 \pm 0.07$ & $0.19 \pm 0.09$ & $0.02^{*}$ \\
DI & $0.17 \pm 0.08$ & $0.24 \pm 0.10$ & $0.00^{*}$ \\
\hline
\end{tabular}

Data are presented as means $\pm \mathrm{SD}$

TDD1 CSII total daily dose (IU/kg/day), Basal CSII basal insulin dose (IU/kg/day), Bolus CSII bolus insulin dose (IU/kg/day), TDD2 thrice-daily total daily dose (IU/ $\mathrm{kg} /$ day), $B I$ breakfast insulin dose (IU/kg), $L I$ lunch insulin dose (IU $/ \mathrm{kg}), D I$ dinner insulin dose (IU/kg) ${ }^{*} p<0.05$

Matsuda indices. Our data showed that male and female patients had similar C-peptide concentrations at $0 \mathrm{~min} \quad(p=0.60), 30 \mathrm{~min}$ $(p=0.62)$ and $120 \mathrm{~min}(p=0.28)$ after bread load (Table 4). We also analyzed the $\beta$-cell function and insulin sensitivity between male and female patients at baseline. Our data showed that male and female patients had similar HOMA-B values $(p=0.14)$. However, male patients had significant increases in the Matsuda Index $(p=0.04)$ and decreases in the HOMA-IR $(p=0.04)$ compared with female patients (Table 4 ).

\section{DISCUSSION}

In this study, we observed that the incidence of hypoglycemia as monitored by CGM was $28 \%$ in patients receiving intensive insulin therapy. Binary logistic stepwise regression analysis showed that only gender was significantly correlated with hypoglycemia. Importantly, our data indicated that male patients had a significantly higher hypoglycemic ratio than female patients, although we observed that male patients with T2D required significantly lower insulin doses to achieve glycemic control and
Table 4 The $\beta$-cell function and insulin sensitivity in male and female patients at baseline

\begin{tabular}{lccl}
\hline Items & Male group & Female group & $\boldsymbol{p}$ value \\
\hline C-peptide 0 & $2.0 \pm 1.0$ & $2.1 \pm 1.0$ & 0.60 \\
C-peptide 30 & $2.7 \pm 1.5$ & $3.3 \pm 2.1$ & 0.62 \\
C-peptide 120 & $4.9 \pm 2.9$ & $5.1 \pm 2.8$ & 0.28 \\
HOMA-B & $19.7 \pm 15.1$ & $22.5 \pm 23.4$ & 0.14 \\
HOMA-IR & $2.6 \pm 1.6$ & $3.4 \pm 2.6$ & $0.04^{*}$ \\
Mastuda Index & $104.3 \pm 87.4$ & $91.7 \pm 63.3$ & $0.04^{*}$ \\
\hline
\end{tabular}

Data were presented as means $\pm \mathrm{SD}$

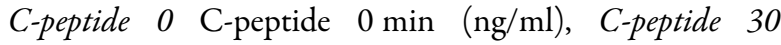
C-peptide $30 \mathrm{~min}$ (ng/ml), C-peptide 120 C-peptide $120 \mathrm{~min}(\mathrm{ng} / \mathrm{ml})$, HOMA-B homoeostasis model assessment B, HOMA-IR homoeostasis model assessment IR ${ }^{*} p<0.05$

significant pre-mixed insulin analog doses to maintain glycemic control compared with female patients in this study. Our data indicated that attention should be paid to preventing hypoglycemia in male patients receiving intensive insulin therapy even at lower insulin doses than in female patients.

Achieving the optimal HbA1c level in patients with T2D is the main target for physicians when choosing glucose-lowering therapies [29]. HbA1c is generated by the exposure of overall blood profiles for the long term, which does not necessarily reflect daily plasma glucose variations over the entire day [30, 31]. CGM provides a unique opportunity to examine the 24-h glucose glycemic excursions in patients with T2D, which might be the best tool to determine the overall blood glucose profiles [4]. Intensive insulin therapy has been used to treat patients with T2D in China [7]. Intensive insulin therapy consists of CSII using an insulin pump and multiple daily injections (MDIs) [32]. Some patients with longstanding type 2 diabetes mellitus respond well to intensive treatment [33] and are able to maintain euglycemia with minimal treatment for a long period [34]. Indeed, patients' response to intensive insulin therapy is quite variable, mirroring the heterogeneity of diabetes [33]. Reports have 
highlighted the importance of hypoglycemia in intensive insulin therapy compared with conventional therapy with or without the use of an insulin pump [13].

Why male patients had a higher incidence of hypoglycemia during intensive insulin therapy is not known. However, this highlights that more careful blood glucose monitoring may be necessary in patients receiving intensive insulin therapy, especially males, to prevent the occurrence of hypoglycemia. The number of older patients with diabetes started on intensive insulin treatment has been increasing in recent years [35]. This has mainly been in T1D. Very few studies have used CSII in older patients with T2D. A relatively large study did highlight the increased risk of hypoglycemia in the older patients $[16,17]$. We further demonstrated that male patients were prone to having hypoglycemia monitored by CGM during intensive insulin therapy [4]. In this study, the men and women had the similar ages, courses of disease, BMIs, waistlines, waist-to-hip ratios and $\mathrm{HbA}_{1 \mathrm{c}}$ values. We could not exclude that other factors might affect the insulin doses in patients with T2D in maintaining glycemic control, such as the age, course of T2D, BMI, HbA1c, waistline, waist-to-hip ratio, HOMA-B, HOMA-IR and Mastuda Index. However, multivariate analysis, controlling for all the above-mentioned factors, showed that gender still was an independent factor affecting the incidence of hypoglycemia between the two groups.

Our data indicated that, as expected, male and female patients had similar pancreatic $\beta$ cell function (HOMA-B). In this study, the BMI of the recruited patients was relatively low $\left(24.37 \pm 4.69 \mathrm{~kg} / \mathrm{m}^{2}\right)$. This was in agreement with Ntuk et al. who reported that Chinese women with BMI $24.0 \mathrm{~kg} / \mathrm{m}^{2}$ and Chinese men with BMI $26.0 \mathrm{~kg} / \mathrm{m}^{2}$ have equivalent prevalences of diabetes at BMI $30 \mathrm{~kg} / \mathrm{m}^{2}$ as white participants [36]. Interestingly, female patients had an insignificant increase in BMI compared with male patients in this study. Thus, we inferred the trend of a decrease in body weight might be the reason for the increased insulin sensitivity, because body fat weight is associated with a decreased Matsuda Index and increased HOMA-IR [28]. The increased insulin sensitivity in male patients compared with female patients indicated that the body fat weight might be related to the Matsuda Index and HOMA-IR. Future studies are needed to identify the differences in insulin resistance between male and female patients.

Our study has other limitations. First, the study only observed a single city in Northern China, and the situation might not be the same in other cities. Second, the sample size was relatively small. Third, our observations were not carried out for a long time period.

\section{CONCLUSIONS}

In conclusion, our data suggested that male patients with T2D required lower insulin doses to maintain glycemic control. Our outcome indicated that increased attention should be paid to preventing hypoglycemia in male patients receiving intensive insulin therapy.

\section{ACKNOWLEDGEMENTS}

We are grateful to Prof. Frank Elliott, who contributed to the final proof of the manuscript.

Funding. This research was funded by the Science and Technology Support Program of Jiangsu Province (China) (no. BL2014010) and the Natural Science Foundation of Jiangsu Province (BK20170136). The article processing charges were funded by the authors.

Authorship. All named authors meet the International Committee of Medical Journal Editors (ICMJE) criteria for authorship for this manuscript, take responsibility for the integrity of the work as a whole and gave final approval for the version to be published.

Authorship Contributions. Jian-hua Ma and Lei Ye contributed to the conception and design of the study. Feng-fei Li, Wen-li Zhang, Xiaomei Liu, Yi-xuan Sun, Mao-yuan Chen and Ying Zhang contributed to the conduct/data collection. Xiao-fei Su and Jin-dan Wu contributed to 
the data analysis. Feng-fei $\mathrm{Li}$ contributed to writing the manuscript and its final approval.

Disclosures. Feng-fei Li, Ying Zhang, Wen-li Zhang, Xiao-mei Liu, Mao-yuan Chen, Yi-xuan Sun, Xiao-fei Su, Jin-dan Wu, Lei Ye and Jianhua Ma have nothing to disclose.

Compliance with Ethics Guidelines. All procedures followed were in accordance with the Helsinki Declaration of 1964 , as revised in 2013. Informed consent was obtained from all patients for being included in the study.

Data Availability. The data sets generated during and/or analyzed during the current study are not publicly available but are available from the corresponding author on reasonable request.

Open Access. This article is distributed under the terms of the Creative Commons Attribution-NonCommercial 4.0 International License (http://creativecommons.org/licenses/ by-nc/4.0/), which permits any noncommercial use, distribution, and reproduction in any medium, provided you give appropriate credit to the original author(s) and the source, provide a link to the Creative Commons license, and indicate if changes were made.

\section{REFERENCES}

1. Esteghamati A, Mousavizadeh M, Noshad S, et al. Gender-dependent effects of metformin on vaspin and adiponectin in type 2 diabetes patients: a randomized clinical trial. Hormone Metab Res. 2013;45(4):319-25.

2. Kautzky-Willer A, Harreiter J, Pacini G. Sex and gender differences in risk, pathophysiology and complications of type 2 diabetes mellitus. Endocr Rev. 2016;37(3):278-316.

3. Glechner A, Harreiter J, Gartlehner G, et al. Sexspecific differences in diabetes prevention: a systematic review and meta-analysis. Diabetologia. 2015;58(2):242-54.

4. Li FF, Liu BL, Zhu HH, et al. Continuous glucose monitoring in newly diagnosed type 2 diabetes patients reveals a potential risk of hypoglycemia in older men. J Diabetes Res. 2017;2740372.

5. Harreiter J, Kautzky-Willer A. Sex and gender differences in prevention of type 2 diabetes. Front Endocrinol. 2018;9:220.

6. Li FF, Fu LY, Zhang WL, et al. Blood glucose fluctuations in type 2 diabetes patients treated with multiple daily injections. J Diabetes Res 2016;1028945.

7. Weng J, Li Y, Xu W, et al. Effect of intensive insulin therapy on beta-cell function and glycaemic control in patients with newly diagnosed type 2 diabetes: a multicentre randomised parallel-group trial. Lancet. 2008;371(9626):1753-60.

8. Retnakaran R, Drucker DJ. Intensive insulin therapy in newly diagnosed type 2 diabetes. Lancet. 2008;371(9626):1725-6.

9. Ilkova H, Glaser B, Tunckale A, et al. Induction of long-term glycemic control in newly diagnosed type 2 diabetic patients by transient intensive insulin treatment. Diabetes Care. 1997;20(9): 1353-6.

10. Li Y, Xu W, Liao Z, et al. Induction of long-term glycemic control in newly diagnosed type 2 diabetic patients is associated with improvement of beta-cell function. Diabetes Care. 2004;27(11):2597-602.

11. Ryan EA, Imes S, Wallace C. Short-term intensive insulin therapy in newly diagnosed type 2 diabetes. Diabetes Care. 2004;27(5):1028-32.

12. Reznik Y, Cohen O, Aronson R, et al. Insulin pump treatment compared with multiple daily injections for treatment of type 2 diabetes (OpT2mise): a randomised open-label controlled trial. Lancet. 2014;384(9950):1265-72.

13. The Diabetes Control and Complications Trial Research Group. The effect of intensive treatment of diabetes on the development and progression of long-term complications in insulin-dependent diabetes mellitus. N Engl J Med. 1993;329(14):977-86.

14. Lv WS, Li L, Wen JP, et al. Comparison of a multiple daily insulin injection regimen (glargine or detemir once daily plus prandial insulin aspart) and continuous subcutaneous insulin infusion (aspart) in short-term intensive insulin therapy for poorly controlled type 2 diabetes patients. Int J Endocrinol. 2013; 614242.

15. The DCCT Research Group. Epidemiology of severe hypoglycemia in the diabetes control and complications trial. Am J Med. 1991;90(4):450-9. 
16. Herman WH, Ilag LL, Johnson SL, et al. A clinical trial of continuous subcutaneous insulin infusion versus multiple daily injections in older adults with type 2 diabetes. Diabetes Care. 2005;28(7):1568-73.

17. Bremer JP, Jauch-Chara K, Hallschmid M, et al. Hypoglycemia unawareness in older compared with middle-aged patients with type 2 diabetes. Diabetes Care. 2009;32(8):1513-7.

18. Li FF, Liu BL, Yin GP, et al. Young onset type 2 diabetic patients might be more sensitive to metformin compared to late onset type 2 diabetic patients. Sci Rep. 2017;7(1):16382.

19. Li H, Li W, Gu Y, et al. Comparison of continual insulin or secretagogue treatment in type 2 diabetic patients with alternate insulin-secretagogue administration. Diabetes Res Clin Pract. 2009;84(2):158-62.

20. Li FF, Zhang WL, Liu BL, et al. Management of glycemic variation in diabetic patients receiving parenteral nutrition by continuous subcutaneous insulin infusion (CSII) therapy. Sci Rep. 2018;8(1):5888.

21. Li FF, Jiang L, Fu L, et al. Exenatide add-on to continuous subcutaneous insulin infusion therapy reduces bolus insulin doses in patients with type 2 diabetes: a randomized, controlled, open-label trial. Diabetes Ther. 2017;8(1):177-87.

22. Li FF, Xu XH, Fu LY, et al. Influence of acarbose on plasma glucose fluctuations in insulin-treated patients with type 2 diabetes: a pilot study. Int J Endocrinol. 2015;903524.

23. Li FF, Shen Y, Sun R, et al. Effects of Vildagliptin add-on insulin therapy on nocturnal glycemic variations in uncontrolled type 2 diabetes. Diabetes Ther. 2017;8(5):1111-22.

24. Li FF, Liu BL, Yan RN, et al. Features of glycemic variations in drug naive type 2 diabetic patients with different HbA1c values. Sci Rep. 2017;7(1):1583.

25. Haahr H, Sasaki T, Bardtrum L, et al. Insulin degludec/insulin aspart in Japanese patients with type 1 diabetes mellitus: Distinct prandial and basal glucose-lowering effects. J Diabetes Investig. 2016;7(4):574-80.

26. Matthews DR, Hosker JP, Rudenski AS, et al. Homeostasis model assessment: insulin resistance and beta-cell function from fasting plasma glucose and insulin concentrations in man. Diabetologia. 1985;28(7):412-9.

27. Matsuda M, DeFronzo RA. Insulin sensitivity indices obtained from oral glucose tolerance testing: comparison with the euglycemic insulin clamp. Diabetes Care. 1999;22(9):1462-70.

28. Sasaki R, Yano Y, Yasuma T, et al. Association of waist circumference and body fat weight with insulin resistance in male subjects with normal body mass index and normal glucose tolerance. Intern Med. 2016;55(11):1425-32.

29. Inzucchi SE, Bergenstal RM, Buse JB, et al. Management of hyperglycemia in type 2 diabetes, 2015: a patient-centered approach: update to a position statement of the American Diabetes Association and the European Association for the Study of Diabetes. Diabetes Care. 2015;38(1):140-9.

30. Nathan DM, Kuenen J, Borg R, et al. Translating the A1C assay into estimated average glucose values. Diabetes Care. 2008;31(8):1473-8.

31. Del Prato S. In search of normoglycaemia in diabetes: controlling postprandial glucose. Int J Obes Relat Metab Disord. 2002;26(Suppl 3):S9-17.

32. Jia W, Xiao X, Ji Q, et al. Comparison of thrice-daily premixed insulin (insulin lispro premix) with basalbolus (insulin glargine once-daily plus thrice-daily prandial insulin lispro) therapy in east Asian patients with type 2 diabetes insufficiently controlled with twice-daily premixed insulin: an openlabel, randomised, controlled trial. Lancet Diabetes Endocrinol. 2015;3(4):254-62.

33. Retnakaran R, Yakubovich N, Qi Y, et al. The response to short-term intensive insulin therapy in type 2 diabetes. Diabetes Obes Metab. 2010;12(1):65-71.

34. Reznik Y, Cohen O, Aronson R, et al. Insulin pump treatment compared with multiple daily injections for treatment of type 2 diabetes (OpT2mise): a randomised open-label controlled trial. Lancet. 2014;384(9950):1265-72.

35. Yeoh E, Beato-Vibora P, Rogers H, et al. Efficacy of insulin pump therapy in elderly patients. Diabetes Technol Ther. 2015;17(5):364-5.

36. Ntuk UE, Gill JM, Mackay DF, et al. Ethnic-specific obesity cutoffs for diabetes risk: cross-sectional study of 490,288 UK biobank participants. Diabetes Care. 2014;37(9):2500-7. 